\title{
Akademisierung der Hausarztmedizin an der Universität Zürich - eine Billanz nach drei Jahren
}

\author{
Nach mehrjährigem politischem Vorspiel wurde im März 2008 das Institut für Haus- \\ arztmedizin (IHAM) am UniversitätsSpital Zürich gegründet, zusammen mit dem \\ gleichnamigen Lehrstuhl an der Universität Zürich. Nach nunmehr drei Jahren soll \\ nachfolgend eine erste Bilanz gezogen werden.
}

\section{Lehre}

Die Hausarztmedizin war bereits vor der Schaffung des Lehrstuhls in die studentische Lehre involviert, allerdings war die Evaluation seitens der Studierenden sehr inhomogen. Basierend auf schriftlichen Befragungen, aber auch auf zahlreichen intensiven Gesprächen mit den Studierenden wurde eine Reihe von Massnahmen umgesetzt. So wurden die hausärztlichen Beiträge didaktisch und inhaltlich überarbeitet. Neu wurde im 6. Jahr ein dreitägiger Blockkurs «Hausarztmedizin» eingeführt, in dem das Fach umfassend dargestellt werden kann. Die Evaluation durch die Studierenden verbesserte sich um über eine Notenstufe und belegt den Erfolg der Massnahmen. Die umfangreichste Innovation in der Lehre war die Einführung des Einzeltutoriates in einer Hausarztpraxis für alle Studierenden.

Im Rahmen der «Bologna-Reform» wurde die Anfertigung einer Masterarbeit für die Studierenden obligat. Mit 24 aktuell betreuten Masterarbeiten und 15 Dissertanden ist das Engagement des IHAM weit überdurchschnittlich. Dieses hohe Engagement ist nicht ohne Kalkül, denn es ermöglicht den frühen und intensiven Kontakt der Studierenden mit hausärztlichen Problemstellungen. Die praxisorientierten Fragestellungen werden von den Studierenden sehr geschätzt, wie die zahlreichen Anfragen eindrucksvoll belegen. So entstehen bereits in einer sehr frühen Phase der medizinischen Ausbildung quantitativ und qualitativ neue Kontakte mit einer selbstbewussten und selbständigen Disziplin.

Eine weitere Möglichkeit des intensiven Kontaktes zwischen Hausarztmedizin und Studierenden bietet das Mentoring-Programm, das neu auch von der SGAM finanziell unterstützt wird. Hier fungieren Hausärzte als Mentoren für Studierende, beraten und begleiten sie über mehrere Jahre hinweg. Das Programm erfreut sich auf beiden Seiten grosser Beliebtheit mit jährlich bis zu 25 Hausärzten als Mentoren.

\section{Chaire de médecine de famille à} I'Université de Zurich - bilan après

\section{trois ans}

La création de I'IHAM peut être considérée comme une réussite: la médecine de famille fait partie intégrante de l'enseignement médical et, avec le tutorat individuel, elle inaugure une des sessions les mieux évaluées de tout le cursus. Le curriculum Médecin de famille de l'Hôpital universitaire de Zurich a permis d'avancer avec succès sur la voie de la consolidation et de la qualification de la relève médicale. La présence de I'IHAM à I'université tout comme la coopération avec les cliniques de l'Hôpital universitaire de Zurich a sensiblement amélioré la perception de la médecine de famille et provoqué une nette reprise de la demande. L'Institut s'est d'emblée hissé à la pointe de la recherche dans les régions germanophones, et ce malgré la situation délicate créée par les moyens tiers. L'évolution observée à Zurich met clairement en évidence le potentiel dont recèlent les instituts universitaires de médecine de famille du point de vue de la promotion de la relève mais aussi au regard du bénéfice scientifique. Dès lors, il faut créer le plus rapidement possible des instituts dotés de ressources financières solides dans toutes les universités suisses, et mettre à disposition des fonds publics tiers débloqués selon des critères visant à stimuler la concurrence, notamment dans l'intérêt d'un système de santé efficace, axé sur le patient. 


\section{Forschung}

\section{Bedarf an hausärztlicher Forschung}

Es gibt zahlreiche Daten, die eindrucksvoll belegen, dass die Hausarztmedizin gerade in den Gesundheitssystemen eine starke Rolle innehat, in denen auch die hausärztliche Forschung etabliert ist. Dies ist im Grunde nicht verwunderlich, denn je mehr eine Profession die eigene Leistung valide belegt, umso mehr wird sie geschätzt. Ein schönes Beispiel sind hier die Niederlande, wo 2005 im Rahmen einer umfangreichen Gesundheitsreform auch die zentrale Stellung des Hausarztes auf dem Prüfstand stand. Durch zahlreiche gute Belege für die Effizienz dieses Systems nahm die Politik davon Abstand, dies zu ändern.

\section{«In der Forschung konnte das}

Institut auf Anhieb eine Spitzenposition im deutschsprachigen

\section{Raum übernehmen.»}

Für die Schweiz - wie auch für zahlreiche andere Länder - gilt noch immer, was in einer Stellungnahme der Spitzenverbände der Krankenkassen (!) in Deutschland an den Sachverständigenrat der Bundesregierung im Jahre 2000 festgestellt wurde:

«Die belastbare Evidenz (...) zur Über-, Unter- und Fehlversorgung ist vor allem deshalb so gering, weil im Bereich der Universitäten ein völlig unangemessenes Missverhältnis zwischen klinischer Forschung und Versorgungsforschung zu verzeichnen ist (...) Damit verbunden ist eine massive Überschätzung und Überbewertung der medizinischen Interventionen für die Lebenserwartung und Lebensqualität und eine nicht zukunftsfähige Vernachlässigung von Forschung zur interdisziplinären und sektorenübergreifenden Gestaltung von Versorgungszusammenhängen.»

Nach wie vor fussen gesundheitspolitische Entscheidungen selten auf einer Evidenz, sondern vielmehr auf Willkür und Lobbyismus. Dies ist ein erstaunlicher Widerspruch - während man auf der Mikroebene der medizinischen Versorgung - also im ärztlichen Alltag - heute mehr denn je eine an Evidenz orientierte Diagnostik und Therapie fordert, orientieren sich gesundheitspolitische Entscheidungen, also die Makroebene, nur marginal an validen Daten; im Wesentlichen eine logische Konsequenz der Tatsache, dass entsprechende Daten schlichtweg fehlen. Denken wir nur an die Selbstdispensation, das Praxislabor oder bald auch die DRGs -Evidenz für das Für oder Wider fehlt. Das müsste nicht so sein, es wäre methodisch möglich, diese Fragestellungen valide zu beantworten. Aus jedem Bereich der medizinischen Versorgung, in dem ein Grossteil der Bevölke- rung primär und in $90 \%$ der Fälle auch abschliessend behandelt wird, fehlen fast gänzlich die Daten. Versorgungsforschung ist somit letztlich auch ein Instrument der Politikberatung, um eine auf Fakten basierende, effiziente, zukunftsfähige Ausrichtung des Gesundheitssystems zu ermöglichen. Rudolf Virchow (1821-1902) formulierte schon vor mehr als hundert Jahren: «Die Medizin ist eine soziale Wissenschaft, und die Politik ist nichts weiter als Medizin im Großen.»

Während in vielen Ländern «Health Services Research» ein prosperierendes Forschungsgebiet ist, existieren hierzulande bislang keine öffentlichen Fördergelder, um diese Art von Forschung voranzutreiben. Ein Antrag der fünf Institute für Hausarztmedizin auf Einrichtung eines Nationalen Forschungsprogramms wurde abgelehnt und stattdessen das Thema «Ressource Holz» gefördert. Solange die Prioritäten in dieser Form gesetzt werden, sieht es für eine wirklich gute Datenlage zum Gesundheitssystem in der Schweiz noch finster aus.

\section{Forschung am IHAM Zürich}

Im IHAM wird derzeit eine Fülle von Projekten durchgeführt. Das reicht von der Evaluation der Notfallpraxis am Waidspital, einem Freiwilligendienst für Sozialbenachteiligte über Cluster-randomisierte Interventionsstudien mit multiprofessionellen Versorgungsansätzen bei Diabetikern (CARAT) bis hin zu diagnostischen Studien und qualitativen Untersuchungen. Im langfristig angelegten FIRE-Forschungsnetzwerk werden über die elektronischen Krankengeschichten Daten über die hausärztliche Versorgung gesammelt. So können aktuell bereits über 308000 Arzt-Patienten-Konsultationen vollumfänglich ausgewertet werden. Darauf basiert auch das SHIFT-Projekt (impact of the Swiss DRG HospItal payment system on the demand For ouTpatient care), das die Auswirkungen der

\section{Im Mentoring-Programm fungieren Hausärzte als Mentoren für Studierende.}

DRGs auf die ambulante Versorgung abbilden wird. Möglich ist dies unter anderem durch eine breitgefächerte Methodenkompetenz. Neben Medizinern arbeiten im Institut auch Epidemiologen, Psychologen, Soziologen, Public-Health-Experten und Pflegefachkräfte. Mit einigen Projekten ist das IHAM in internationale Forschungsverbünde eingebunden, so etwa in das «EurOOHnet», das den «Out-of-hoursService», also den hausärztlichen Notfalldienst, europaweit vergleicht und untersucht.

Die internationale Verknüpfung hat auch zu einem gemeinsamen PhD-Programm mit der Universi- 
In jedem Semester nehmen 220 Lehrärzte Studenten in ihrer Praxis auf. Zudem gibt es ein Mentoring-Programm, in dem Hausärzte die Studierenden über mehrere Jahre hinweg begleiten.

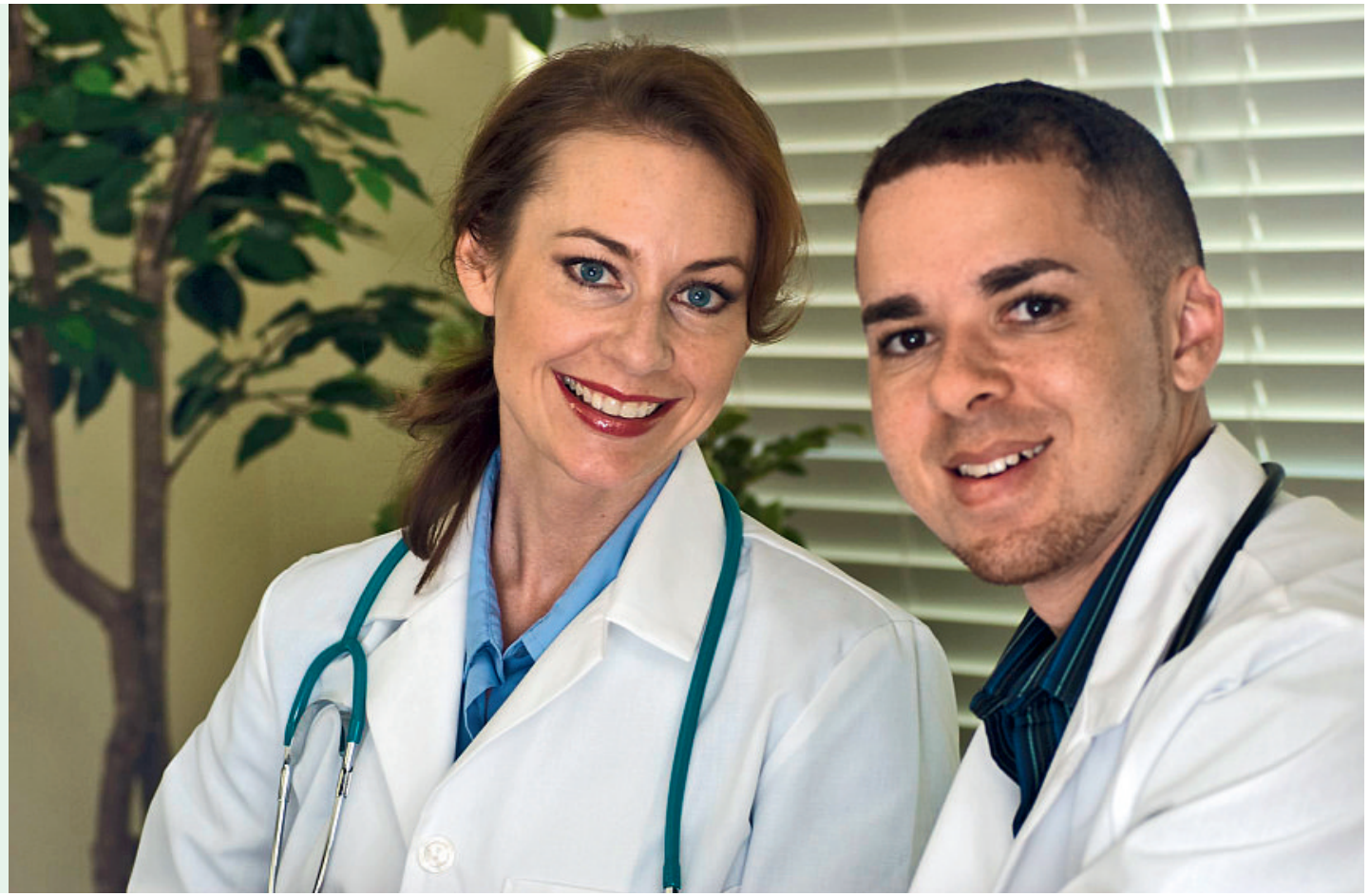

tät Nijmegen/NL und einer gemeinsamen «Summer school on research» geführt, in der sich Schweizer Nachwuchsforscher mit Kollegen aus anderen Ländern zu methodischen Aspekten austauschen und vernetzen können.

Gerade für ein kleines und junges Institut ist es jedoch zentral, sich in der Forschung zu fokussieren und ein eigenes Kompetenzprofil zu entwickeln. Der Fokus des IHAM liegt hier auf den chronischen Erkrankungen, da sie die zentrale medizinische, aber auch ökonomische Herausforderung des 21. Jahrhunderts darstellen. Zudem erfordern gerade sie die klassischen Qualitäten hausärztlicher Betreuung: Kontinuität und ganzheitliche Betreuung statt der Fokussierung auf ein einzelnes Organ, (Sekundär-) Prävention, vertrauensvolle Begleitung und Lotsenfunktion. Und gerade hier eröffnet sich ein enormes Effizienzpotential durch die Optimierung der Versorgungsabläufe.

Sehr erfreulich ist die gute Zusammenarbeit mit anderen Kliniken des UniversitätsSpitals (USZ). So werden einige Studien direkt in Kooperation durchgeführt, in anderen Projekten liefern die Spezialkliniken stets engagiert ihren Input. Teilweise sind die Kliniken sogar aktiv auf das IHAM zugegangen, so etwa bei der diagnostischen Studie minSKIN, mit der die diagnostische Kompetenz von Hausärzten bei malignen Hautveränderungen in Zusammenarbeit mit der dermatologischen Klinik des USZ weiter verbessert werden soll.

Insgesamt ist der wissenschaftliche Output des IHAM beachtlich: Im Jahre 2010 wurden 81 Publika- tionen erstellt, der Grossteil davon wurde in Journals mit respektablem Impactfaktor akzeptiert und publiziert. Damit ist das IHAM im deutschsprachigen Raum das quantitativ und qualitativ erfolgreichste Institut, obgleich die Ressourcen nur einen Bruchteil derer grosser Institute etwa in Deutschland betragen. Ein schöner Beweis Schweizer Effizienz. Ebenso erfreulich ist die Tatsache, dass sich bereits zwei Kollegen am IHAM für das Fach «Hausarztmedizin» habilitieren konnten. Das Ziel, lokalen akademischen Nachwuchs zu generieren, wird also konsequent und erfolgreich umgesetzt.

\section{Forschungsförderung}

Unterstützt wird die Forschung - neben der RRMA («Recherche et réalisation en médecine appliquée»), der Schweizerischen Akademie der Medizinischen

\section{Lehrärzte nehmen}

\section{jedes Semester Studierende} in ihre Praxen auf.

Wissenschaften SAMW, die eine wichtige Quelle darstellt, derzeit praktisch ausschliesslich durch Drittmittel aus Industrie und Stiftungen. Seitens der Universität besteht ein Betriebsmittelkredit von derzeit 29000 Franken. Von den 63 Millionen Franken, die die Universität jährlich dem USZ für Forschung und 
Lehre zur Verfügung stellt, erhält das IHAM aus ungeklärten Gründen nichts. Die Förderung durch die Industrie ist sehr inhomogen.

Während Novartis neben einzelnen Studien sogar eine PhD-Stelle finanziert, unterstützen Menarini, MSD oder Sysmex spezifische Projekte. Die genannten Firmen zeigen sich auch einer kritischen Hausarztmedizin gegenüber offen. Dass dies nicht selbstverständlich ist, zeigten die Erfahrungen mit einem Unternehmen, das auf Antidementiva und Antidepressiva spezialisiert ist: Die Firma rückte von der Absicht, das IHAM zu unterstützen, sofort ab, als ein allgemein gehaltener, kritischer Beitrag des IHAM zu diesen Substanzen erschien. Dies unterstreicht, wie wichtig industrieunabhängige Fördermittel gerade für die Hausarztmedizin sind.

\section{Weiterbildung}

Eine aktuelle Studie des IHAM mit 318 Assistenten, die sich in der Weiterbildung in Innerer Medizin bzw. Chirurgie befinden, zeigte, dass $45 \%$ dieser Kolleginnen und Kollegen grundsätzlich ein Interesse an der Hausarztmedizin hätten bzw. haben. Wir werten dies als Anhaltspunkt dafür, dass das Potential für das Fachgebiet durchaus grösser ist, als dies manche Zahlen zeigen, die von nur $10 \%$ bei Uni-Abgängern sprechen. So wichtig ein früher Kontakt der Studierenden

\section{«Das IHAM versteht sich als die akademische 〈Heimat〉 und Reprä- sentanz der Hausärzteschaft.»}

mit dem Fach Hausarztmedizin ist, so wichtig ist es auch, dieses Potential in der Weiterbildung zu nutzen. Denn gerade in der Weiterbildungsphase gibt es einen substantiellen Nachteil für die Hausarztmedizin: Um eine wirklich qualitativ hochstehende Weiterbildung zu erhalten, muss der Nachwuchs mühsam einzelne Spezialfächer aneinanderreihen und mehrmals die Stelle wechseln. In Verhandlungen mit der Gesundheitsdirektion Zürich konnte erreicht werden, dass am 1. Januar 2010 vier Assistentenstellen am USZ für das IHAM eingerichtet wurden. Mit den Spezialkliniken wie Dermatologie, ORL, Augen und Rheumatologie wurde dann vereinbart, sich die Kosten der Curriculumsassistenten zu teilen, so dass potentiell bis zu sieben Stellen vergeben werden können. Davon profitieren alle Beteiligten: Die Assistenten reihen nahtlos drei Spezialitäten aneinander, um dann das letzte Jahr in einer Hausarztpraxis zu verbringen, und die Kliniken haben stets engagierte und motivierte Assistenten. Regelmässige Mentoringgespräche sichern zudem, dass wirklich die hausärztlich relevanten Inhalte vermittelt werden. Sehr erfreulich ist die rege Nachfrage nach dem Curriculum: Alle Stellen sind besetzt und die Nachfrage ist deutlich grösser als das Angebot.

\section{Positionierung in der Fakultät und dem USZ}

Neben dem Lehrstuhl werden derzeit 250 Stellenprozente für Wissenschaftliche Mitarbeiter und eine Sekretariatsposition seitens der Universität finanziert. Das USZ stellt die Infrastruktur zur Verfügung. Durch die ständig wachsende Zahl der Mitarbeiter, zurzeit 14, musste das IHAM bereits zweimal umziehen und ist nun gemeinsam mit dem Horten-Zentrum (Leiter: Professor Steurer) in der Pestalozzistrasse in der Nähe des USZ untergebracht. Eine enge Zusammenarbeit besteht mit der Medizinischen Klinik und Poliklinik (Professor Battegay): So rotiert jeweils ein Assistent für ein halbes Jahr in die Forschung der Hausarztmedizin. Dieser Austausch hat nicht nur bereits guten wissenschaftlichen Ertrag generiert, er hat sich zudem auch als zusätzlicher Katalysator für den Weg in die hausärztliche Praxis erwiesen.

\section{Zusammenarbeit mit der Hausärzteschaft}

Das IHAM versteht sich als die akademische «Heimat» und Repräsentanz der Hausärzteschaft. Eine enge und vertrauensvolle Zusammenarbeit geniesst allein schon deshalb oberste Priorität und ist letztlich erst die wirkliche Legitimation für den universitären Vertretungsanspruch. Die Zusammenarbeit erfolgt bislang auf zahlreichen Ebenen und mit verschiedenen Schnittstellen. Neben dem Netz von 220 Lehrärzten, die jedes Semester die Studierenden in ihren Praxen aufnehmen, beteiligen sich regelmässig Hausärzte mit grossem Engagement an den zahlreichen Studien und Forschungsprojekten. Seit längerem existiert auch eine «Kerngruppe Forschung», in der praxisrelevante Themen diskutiert und entwickelt werden. Schnittstellen existieren weiter über den Lehrärztetag und zwei Hausarztnachmittage, die in Zusammenarbeit mit der Medizinischen Klinik und Poliklinik sowie dem Verein Hausärzte Zürich (VHZ) organisiert werden.

\section{Resümee}

Die positiven Impulse, die vom IHAM Zürich für die Hausarztmedizin ausgehen, sind evident. Gerade im internationalen Vergleich sind die Ressourcen immer noch extrem bescheiden. Und auch die aktivsten Institute können nicht ersetzen, was die Hausarztmedizin am dringendsten braucht: ein klares Bekenntnis der Politik und Taten statt Worte. 